\title{
Application of an environmental impact assessment methodology for areas exhibiting enhanced levels of NORM in Norway and Poland
}

\author{
A. Hosseini ${ }^{1}$, J.E. Brown ${ }^{1}$, M. Szymańska ${ }^{2}$ and K. Ciupek ${ }^{2}$ \\ ${ }^{1}$ Norwegian Radiation Protection Authority, P.O. Box 55, N-1332 Østeras, Norway. \\ ${ }^{2}$ Central Laboratory for Radiological Protection.03-194 Warszawa, Konwaliowa St. 7, Poland
}

\begin{abstract}
During the last decade or so, the topic of environmental protection from ionising radiation has become a major issue within the field of radiological protection. The change of focus from a purely anthropocentric approach has led to the development and availability of bespoke methodologies for the assessment of impacts on wildlife from radioactive contaminants. The application of such assessment methodologies to actual situations, however, remains relatively limited. This paper describes the implementation of the ERICA Integrated Approach and associated tools to areas exhibiting elevated levels of NORM at Søve in Norway and Wislinka and Kaniow in Poland. In all cases, lichen and bryophytes were found to be the most exposed organisms and tree and large mammals the least exposed. With the exception of Kaniow, Ra-226 is the radionuclide that dominates in terms of its contribution to the total dose rates for all animal organism groups. For plants, Th-228 and Po-210 are dominant contributors. While Th-228 is the primary contributor to external dose rates for all species at Søve and Wislinka, Ra-226 is the primary contributor to internal dose rates in most cases for all sites.
\end{abstract}

\section{INTRODUCTION}

In order to assess the radiation risks associated with existing situations and potential remediation measures, the focus in most countries has been upon human radiological protection. This in turn reflects underpinning legislative requirements and criteria in these countries. Nonetheless, and in view of the International Commission on Radiological Protection's recently stated aims [1] with respect to environmental protection, it seems increasingly likely that more countries, beyond the handful already doing so, will need to address issues related to environmental risks from radiation exposure. In this respect, it was deemed useful to apply an existing environmental risk assessment methodology to selected sites with the aim of exploring the limitations and efficacy of the selected approach. The selected sites for the present study are areas with waste containing elevated levels of Naturally Occurring Radioactive Materials (NORM) as a result of mining activities in the past. Although these areas are reasonably well characterised in terms of underlying geology and the industrial processes undertaken at the sites, they have not been fully mapped with respect to the type of plants and animals that might be living there. To deal with this limitation, a generic approach has been adopted in the present work.

\section{SITE DESCRIPTION}

\subsection{Søve}

The Søve site, a disused mining complex in Telemark County Norway, has been previously identified as a potential radiological hazard (for humans) and constitutes a legacy site where remediation options are being evaluated. The site is rich in the rock type sovite (named after the area) - a type of calcite rock. At Søve, uranium is associated mainly with the mineral Pyrochlore (a constituent of Sovite). In some locations at the site pyrochlore contains $15-20 \% \mathrm{U}_{3} \mathrm{O}_{8}$. Uranium and uranium decay series radionuclides were a bi-product of the ore treatment activities which focussed on the extraction of niobium. In addition 
to uranium, enhanced levels of thorium decay series nuclides and heavy metals, in particular arsenic are known to occur in the rocks from this area and have been discarded as bi-products of mining operations. From a geographical perspective, 3 main areas can be identified with 3 different types of radioactive wastes [2]: Sludge disposal site, Slag heap and Wash house soils. The latter site is selected for further investigation in the present work. At the wash house tailing site, fractions of the produced niobium minerals were lost during processing and subsequently mixed into the local soil. This tailing site shows elevated levels of $\mathrm{U}$ and Th. The top layer consists of sandy materials of explosion-pulverised stone (from wall-rocks) and crushed concrete originating from the preliminary covering and improvement operations conducted when the mine was decommissioned. Removal of this surface material results in an increase in dose-rate.

\subsection{Wislinka disposal dump}

Phosphogypsum is a waste material of the phosphoric acid production process in the fertilizer industry. One of the largest Phosphogypsum disposal sites in Poland is located in the area Żutawy Gdańskie in northern Poland, near the village of WiIJlinka approximately 12 kilometers east of the regional capital Gdańsk. The area used for the Phosphogypsum stack is a former clay pit near a brickyard that has been inactive for over 30 years. The Phosphogypsum was transported to the Wislinka disposal dump from the Gdansk Phosphate Fertilizer Plant "Fosfory" which was in operation from 1972 until the end of 2009. The disposal site covers an area of 36 hectares, 26 of which constitute the stack itself, consisting of 16 million tons of Phosphogypsum. The height of the stack ranges between 35 and 40 meters. The stack is currently covered by dry sewage sludge on which pigweed is grown to protect the surface against wind erosion. The Phosphogypsum stack has been the subject of numerous research studies due to the presence of radioactive isotopes and chemical compounds that may pose a risk to human health and the local environment. Analyses of surface water, groundwater, air, soil and biota around the site have been undertaken.

\subsection{Kaniow}

During the exploitation of hard coal reserves in Poland, a system of settling ponds are often employed to allow suspended materials, carried out of the mines by underground brines, to settle before discharge of the water to rivers. In many cases such settling ponds perform a role as a retention reservoir in order to control the volume of brine discharged to rivers. When radium bearing waters are found in a mine, a process of co-precipitation of radium and barium (as radium-barium sulphate) can occur which leading to elevated concentrations of radium in bottom sediments. The settling pond of concern in this case, Kaniów, is associated with a coal mine in which radium bearing waters are found. This pond was specially constructed as a settling and retention pond to protect the fresh waters of the Vistula River, the ultimate receiving body for the discharge of mine waters. The Kaniów pond replaced an old settling pond, Rontok Wielki, which was a small natural lake but is now contaminated by sediments with high concentrations of radium. The Kaniów pond functions to act as a reservoir for sediments and to protect against the uncontrolled release of contaminated sediments. The total surface area of the pond is almost 4.5 ha. The bottom and banks of the pond are sealed by special waterproof membranes and structurally reinforced by concrete blocks. The banks of the pond are overgrown by different species of plant including Polygonum L., Calamagrostis Adans. and Atriplex L.. The pond is located on territory belonging to the mine itself and access to it is limited to staff of the mine.

\section{METHODOLOGY}

In ERICA, environmental risk is quantified by calculating exposure for non-human biota using data on environmental transfer and dosimetry. Calculated exposures are then compared at the screening tiers to 
Table 1. Average activity concentrations in soil for study sites in Norway and Poland (Bq/kg, dry weight).

\begin{tabular}{|l|c|c|c|}
\hline \multirow{2}{*}{ Radionuclide } & Norway & \multicolumn{2}{|c|}{ Poland } \\
\cline { 2 - 4 } & Søve* $^{*}$ & Wislinka & Kaniow \\
\hline U-238 & 5700 & 34 & \\
\hline U-234 & & 42 & \\
\hline Ra-226 & 5560 & 25 & 7260 \\
\hline Pb-210 & 5730 & 102 & 736 \\
\hline Po-210 & 5730 & 118 & 736 \\
\hline Th-232 & 8033 & 22 & 4220 \\
\hline Ra-228 & 9480 & 22 & 4220 \\
\hline Th-228 & 9620 & 33 & 6330 \\
\hline
\end{tabular}

*Soil data used for this assessment are taken from [7]. Entries in italic indicate assumed values.

a predicted no effect dose rate in order to remove from further investigation those situations where the environmental impact is likely to be of negligible concern. The necessarily large datasets underpinning this assessment approach has led to the development of a supporting computer-based tool - the ERICA Tool [3].The ERICA Tool has been used to perform all risk assessment calculations within this case study. An initial step in the type of environmental risk assessment employed by the ERICA Integrated Approach involves "problem formulation" or defining the assessment context in order to guide further analyses. A key element within the problem formulation stage is the identification of the organism(s) for which the impact should be estimated. A simplified approach is to define "reference organisms". The ERICA Tool provides default lists of reference organisms for each of the following three ecosystems: freshwater, marine and terrestrial. These lists cover, at a generic level, the main types of plants and animals that might be reasonably expected to be encountered within a given ecosystem and further incorporates species currently protected by European legislation.

Once the problem formulation stage is complete the assessor can move onto the assessment stage of the analysis which in the ERICA Integrated Approach is organised into three separate tiers, from conservative screening tiers (Tiers 1 and 2) to a more elaborated tier in which data can be analysed probabilistically. The tiered approach is described in more detail elsewhere [3, 4]. For this study, a Tier 2 (screening) assessment has been applied because the functionality at this tier lends itself most appropriately to the requirements of the assessment. Of additional note is the choice of radiation weighting factors which have been provisionally set, by default in the ERICA Tool as 3 for beta radiation and 10 for alpha radiation. For $\alpha$-radiation weighting factors, this is consistent with the upper bound on the range of variation for this factor with regards to population relevant deterministic endpoints as reported by [5]. In consideration of the application of default screening criteria, the (incremental) dose rate screening value selected for this assessment was $10 \mu \mathrm{Gy} \mathrm{h}^{-1}$. This corresponds to the benchmark used within the initial tiers of the ERICA Integrated Approach with the goal to ensure that generic ecosystems (freshwater, marine and terrestrial) should be protected from effects on structure and function under chronic exposure to radionuclides. The proposed $10 \mu \mathrm{Gy} \mathrm{h}^{-1}$ incremental screening dose rate has been derived from examination of data on effects of ionising radiation in non-human biota collated in the ERICA effects database (FREDERICA) as described in [6].

\subsection{Soil input data}

Soil concentrations for relevant radionuclides were employed to conduct a Tier 2 assessment within the ERICA Assessment Tool. All Soil data used for the present case study are shown in Table 1. There were relatively fewer data points for the site in Norway compared to the sites in Poland. Secular equilibrium was assumed to exist between U-238 and Ra-226, Th-232 and Ra-228 and also Pb-210 and Po-210. 


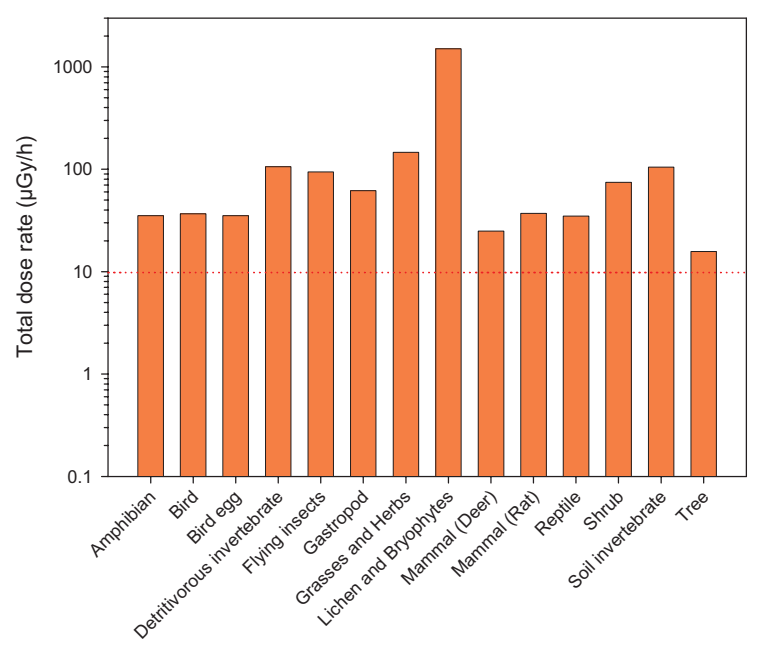

Figure 1. Total weighted dose rates to default reference organisms calculated based on activity concentration in soil for Søve (Wash house area). The dotted line represents the ERICA screening level.

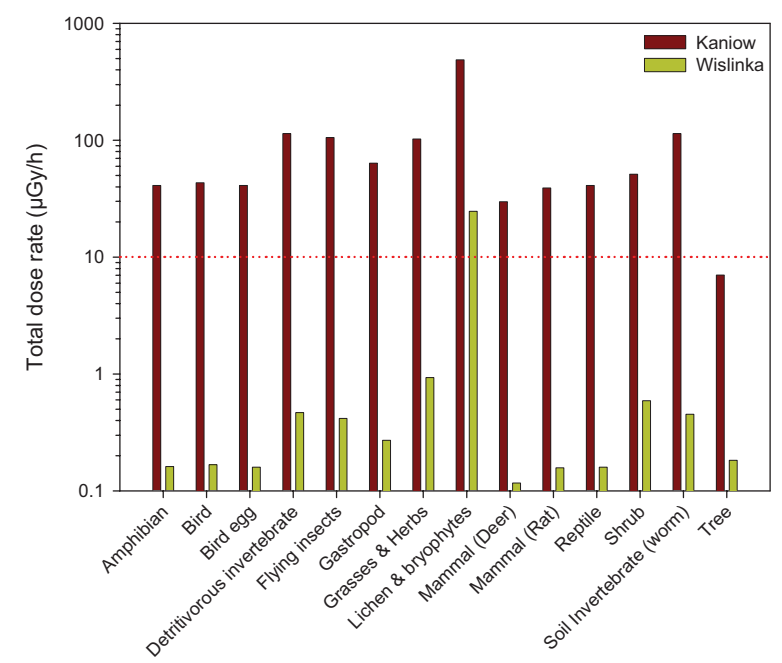

Figure 2. Total weighted dose rates to default reference organisms calculated based on activity concentration in soil for Wislinka and Kaniow sites. The dotted line represents the ERICA screening level.

Furthermore, where there was a lack of data, transient equilibrium between Ra-228 and Th-228 was assumed.

\section{RESULTS}

Best estimate, weighted dose rates to the default suite of ERICA reference organisms based on mean activity concentrations in soil (see Table 1) are presented in Figs. 1 and 2.

As shown in Figs. 1 and 2, Lichen and Bryophytes receive the highest total dose-rate in all sites. The internal component of dose rate dominates almost in all cases and it is Lichen and Bryophytes which receive the highest internal dose. 
The reference organisms which receive the highest external dose differ between the sites. These are Deer, Tree and Rat for Søve (Wash house), Kaniow and Wislinka, respectively. With the exception of Kaniow, Ra-226 is the radionuclide that dominates in terms of its contribution to the total dose rates for animal. For plants Th-228 and and Po-210 are dominant contributors. While Th-228 is the primary contributor to external dose rates for all species at Søve and Wislinka, Ra-226 is the primary contributor to internal dose rates in most cases at all sites.

Based upon the results of the ERICA Assessment Tool, it appears that some species present at the studied sites are potentially being exposed to dose rates which fall above a level where impacts may be confidently considered as negligible, i.e. dose rates exceed the screening bench-mark in numerous cases. This is especially the case for the Søve (Wash house) and Kaniow sites. This does not mean, however, that detrimental effects are occurring but, following the guidance provided by the ERICA integrated approach, necessitates a more detailed assessment to establish whether harmful environmental impacts are likely. In view of this, the assessment for these sites could proceed to the Tier 3 where, by employing more site specific information, a probabilistic analysis of data is possible and this in turn allows for useful statistical information such as the range of exposures to be explored more fully. This further analysis is the subject of future work.

\section{DISCUSSION}

Application of the ERICA Tool to these sites has been a relatively straight forward process as the data entry requirements are highly compatible with much of the information to hand. However, there are conspicuous limitations in the current approach primarily relating to the lack of practicability in using direct measurements of dose-rates in air and uncertainties regarding organism dose-rates arising from the presence of ${ }^{222} \mathrm{Rn}$ and ${ }^{220} \mathrm{Rn}$ in air. Although ambient dose equivalent measurements are not directly comparable (because they are based on human dosimetry) with the calculated external dose rates made by ERICA, they still may provide us with an indication of external absorbed dose-rates which might be experienced by various wild-life categories. Ambient dose equivalent data for Søve [7] were compared with the external dose rates calculated by ERICA. The calculated values were higher by one order of magnitude on average.

Another potential problem is associated with the 10 day cut-off specified as default in ERICA for which Dose Conversion Coefficients (DCC) of progeny are combined with the parent DCC when the physical half-life is below this value. The 10 days cut-off does not allow for the consideration of disequilibrium between parent and daughter radionuclides within particular decay chains. For example ERICA assumes that daughter radionuclides such as ${ }^{222} \mathrm{Rn},{ }^{218} \mathrm{Po},{ }^{214} \mathrm{Po},{ }^{214} \mathrm{Bi}$ and ${ }^{214} \mathrm{Po}$ are all in equilibrium with their parent ${ }^{226} \mathrm{Ra}$ as all of them have a half life of less than 10 days. But analytical measurements from Kaniow show that ${ }^{214} \mathrm{~Pb}$ and ${ }^{214} \mathrm{Bi}$ exhibit much higher concentrations than ${ }^{226} \mathrm{Ra}$.

In all assessments Lichen and Bryophyte show the highest dose rates. According to information provide by [8] on acute lethal doses of radiation, mosses and lichen constitute one of the more radioresistant wild-life groups requiring exposures orders of magnitude higher than those of sensitive groups such as mammals and birds to cause death. What this simply means in the context of this study is that although Lichen and Bryophyte are the most exposed groups they are not necessarily the most at risk. The United Nation Scientific Committee on the Effects of Atomic Radiation [8] have not altered the view from their previous comprehensive report of 1996 that chronic dose rates of less than $100 \mu \mathrm{Gy} / \mathrm{h}$ to the most highly exposed individuals would be unlikely to have significant effect on most terrestrial communities. Weighted absorbed dose rates for most of the wild-life groups analyses in this study fall below this level at all sites.

Furthermore, it is a debatable point whether the prediction of the relatively elevated exposures for Lichen and Bryophyte is, in any case, an artefact. These relatively elevated exposures can probably be explained by considering the derivation and applicability of CRs. $\mathrm{Pb}-210$ and ${ }^{210} \mathrm{Po}$ are primarily introduced to these organisms via surficial deposition after the parent isotope of ${ }^{222} \mathrm{Rn}$ is exhaled 
from soil over a larger region and decays (with a half-life 3.8 days) to these radionuclides via several very short lived intermediates. Evidence for this can be cited from the experimental work of [9] who established that for vegetation with large surface area over ground parts, the contribution from atmospheric deposition for the total activity of these radionuclides could be large. Although they studied grass similar arguments concerning the importance of interception can be forwarded for Lichen and Bryophytes. This has the implication that the measured activity concentration of ${ }^{210} \mathrm{~Pb}$ and ${ }^{210} \mathrm{Po}$ in soil has a rather tenuous indirect relationship to the activity found in (or more probably 'on') these organisms. The applicability of the ERICA default $\mathrm{CR}$ values for ${ }^{210} \mathrm{~Pb}$ and ${ }^{210} \mathrm{Po}$ in lichen and bryophyte, derived from measurements in soil and this wild-life group at predominantly natural low background settings, is questionable and may result in over-predictions for activity concentrations of these radionuclides at technologically enhanced NORM sites (because although soil concentrations are relatively high there is no reason to expect similarly elevated concentrations in lichen and bryophytes as their levels reflect deposition). This indeed appears to be borne out at the Søve site where preliminary (unpublished) measurements of ${ }^{210} \mathrm{~Pb}$ and ${ }^{210} \mathrm{Po}$ in mosses are much lower than values that would have been predicted using soil data and default $\mathrm{CR}$ values.

\section{Acknowledgments}

This work was supported by a grant from Norway through the Norwegian Financial Mechanism and forms part of the PORANO (Survey of the impact of enhanced natural radioactivity on human and natural environments) project, contract no. PNRF-192-AI-1/07. The financial support of the Polish - Norwegian Research Fund is gratefully acknowledged.

\section{References}

[1] ICRP. The 2007 Recommendations of the International Commission on Radiological Protection. ICRP Publication 103. Ann. ICRP 2007; 37 (2-4).

[2] NGI Report. Kartlegging av omfang og kostnader ved eventuell senere opprydning av radioaktivt materiale ved Søve gruver (in Norwegian). (2009) 20091927-00-14-R

[3] Brown J.E., Alfonso B., Avila R., Beresford N.A., Copplestone D., Pröhl G. J. Environ. Radioact. 99 (2008) 1371-1383.

[4] Larsson C.M. J. Environ. Radioact. 99 (2008) 1364-1370.

[5] Chambers, D.B., Osborne, R.V., Garva, A.L. J. Environ. Radioact. 87 (2006) 1-14.

[6] Garnier-Laplace J., Copplestone D., Gilbin R., Alonzo F., Ciffroy P., Gilek M., Agüero A., Björk M., Oughton D.H., Jaworska A., Larsson C.M., Hingston J.L. J. Environ. Radioact. 99 (9) (2008) 1474-1483.

[7] IFE Report. Radiologisk kartlegging av området rundt tidligere Søve gruver (in Norwegian). (2006) IFE/KR/F-2006/174.

[8] UNSCEAR 2008. Report to the General Assembly with Scientific Annexes. VOLUME II, Annex E. Effects of ionizing radiation on non-human biota. United Nations, New York. pp. 97

[9] Pietrzak Flis, Z., Skowrońska-Smolak, M., Science of the Total Environment, 162 (1995) 139-147. 